Published in final edited form as:

Thromb Res. 2014 November ; 134(5): 952-956. doi:10.1016/j.thromres.2014.08.009.

\title{
Platelet Activation Increases in Patients Undergoing Vascular Surgery
}

\author{
Gabriel S. Schneider, MD¹, Caron B. Rockman, MD², and Jeffrey S. Berger, MD ${ }^{1,2,3}$ \\ ${ }^{1}$ Department of Medicine, Division of Cardiology, New York University School of Medicine, New \\ York, NY \\ ${ }^{2}$ Department of Surgery, Division of Vascular Surgery, New York University School of Medicine, \\ New York, NY \\ ${ }^{3}$ Department of Medicine, Division of Hematology, New York University School of Medicine, New \\ York, NY
}

\section{Abstract}

Background-Platelets are a major contributor to atherothrombosis and may contribute to the heightened risk of perioperative cardiovascular events. We sought to examine changes in platelet activity in subjects undergoing vascular surgery.

Methods-Platelet activity in 18 patients (median age 74, $45 \%$ female) undergoing nonemergent open vascular surgery was assessed by light transmission aggregometry in response to saline, epinephrine and adenosine-5 diphosphate (ADP), and by flow cytometric analysis of monocyte-platelet aggregation (MPA). Platelet activity was assessed preoperatively (T1), 1-hour into the operation (T2), 1-hour (T3), 24-hours (T4) and 48-hours post-operatively (T5). Data were compared using the Wilcoxon Signed Ranks Test. Continuous variables are summarized as medians and (interquartile, IQR) ranges.

Results-Spontaneous platelet aggregation increased transiently during the surgical period (T1-5.8\% [2.4, 10.8], T2-13.5\% [9.3, 26.5], T3-7.5\% [3.3, 17], T4-10.0\% [7.3, 16.3], T5-7.25\% [4.5, 29.9], $\mathrm{P}=0.002)$. Similar trends in perioperative platelet activity were noted for platelet aggregation in response to epinephrine $(\mathrm{P}=0.035)$ and $\mathrm{ADP}(\mathrm{P}=0.036)$. Using flow cytometry, we found an increase in MPA during the perioperative period $(\mathrm{P}=0.047)$, which was most significant between $\mathrm{T} 1$ and $\mathrm{T} 3(\mathrm{P}=0.005)$.

Conclusions-Platelet activity increases significantly during and following open vascular surgery. This data may help explain the pathophysiology of increased thrombotic risk during the perioperative period of vascular surgery.

Corresponding author: Jeffrey S. Berger, MD, MS, Assistant Professor of Medicine and Surgery, Director of Cardiovascular Thrombosis, Marc and Ruti Bell Program in Vascular Biology, New York University School of Medicine, 530 First Avenue, Skirball 9R, New York, NY 10016, Tel. 212-263-4004, Fax. 212-263-3988, jeffrey.berger@nyumc.org.

Publisher's Disclaimer: This is a PDF file of an unedited manuscript that has been accepted for publication. As a service to our customers we are providing this early version of the manuscript. The manuscript will undergo copyediting, typesetting, and review of the resulting proof before it is published in its final citable form. Please note that during the production process errors may be discovered which could affect the content, and all legal disclaimers that apply to the journal pertain. 


\section{Introduction}

Subjects undergoing open vascular surgery are at significant risk for perioperative cardiovascular events. ${ }^{1}$ Atherothrombosis is the underlying mechanism of adverse cardiovascular events, and is believed to play a major role in the heightened perioperative risk of cardiovascular complications. ${ }^{2-4}$ Platelets are a major contributor to atherothrombosis and are a key target of intervention in the treatment and prevention of cardiovascular morbidity and mortality in both the operative and non-operative settings. Platelet activity is increased in subjects with peripheral artery disease and vascular surgery may increase platelet activity even further. To date, studies measuring platelet activity in the perioperative period have found conflicting results. ${ }^{5,6}$ While several studies found that platelet aggregation is increased in the early postoperative period, these studies used a limited number of platelet markers. ${ }^{6-8}$

Increased platelet activity is associated with significant morbidity and mortality. ${ }^{9-11}$ Understanding the dynamic profile of platelet activity in the perioperative setting is crucial to understanding the increased risk of cardiovascular events in the perioperative period. We therefore designed a prospective pilot study to evaluate the changes in markers of platelet activity in subjects undergoing non-emergent open vascular surgery.

\section{Methods}

\section{Participants}

Between December 2010 and February 2011, 18 consecutive patients scheduled for open vascular surgery procedures were recruited for the study. Included operations consisted of carotid endarterectomy, open aneurysmal repair and lower extremity bypass repair. Subjects with known thrombocytopenia (platelet count <75), renal failure (creatinine clearance $<30 \mathrm{ml} / \mathrm{min}$ ), active cancer, active infection, predisposition to bleeding, any use of nonsteroidal anti-inflammatory drug within 72 hours, or any antithrombotic therapy were excluded. All participants provided informed consent before participation in the study approved by the local institutional review board.

\section{Study Design}

After informed consent was obtained, blood sampling was performed at set time points during the perioperative period; 1 hour preoperatively (Time point [T] 1), 1-hour into the operation (T2), 1-hour post-operatively (T3), $\approx 24$-hours (1-day) postoperatively (T4), and $\approx 48$-hours (2-days) postoperatively (T5). All samples collected were in the fasting state and samples from T4 and T5 were drawn in the early morning. All patients had an arterial line available for blood collection at T1 to T3. For samples from T4 and T5, blood collection was performed through an arterial line or through venipuncture using a 19-guage needle if the arterial line was already removed. The validity of combining arterial and venous sampling is supported by several studies, including a recent paper from our group that found very good agreement between arterial and venous samples with respect to markers of platelet activity. ${ }^{12}$ For all samples, the first $2 \mathrm{ml}$ was discarded and the remaining blood was collected into a vacutainer tube containing EDTA for complete blood count (CBC) and 
vacutainers containing 3.2\% (0.105 moles/liter) sodium citrate (Becton Dickenson, Franklin Lakes, NJ) for platelet activity. The time between blood draw and aggregometry was uniform at each time point as samples were taken directly to the lab for analysis after collection.

Platelet and hematological parameters-Complete blood count was performed using a Sysmex (Mundelein, Illinois, USA) XE-2100 analyzer. Complete blood count was performed within 60 minutes of phlebotomy.

Light Transmission Aggregometry-Following phlebotomy, blood was centrifuged at $200 \mathrm{~g}$ for 10 minutes to obtain platelet rich plasma (PRP). ${ }^{13}$ Platelet poor plasma (PPP) was obtained by centrifuging PRP at $2500 \mathrm{~g}$ for 10 minutes. Light transmission aggregometry (LTA) was performed according to the manufacturer's specification using Helena (Beaumont, TX, USA) AggRAM light transmission aggregometer, reagents, cuvettes, and stir bars. As previously described, $225 \mathrm{uL}$ of PRP was incubated at $37^{\circ} \mathrm{C}$ for 90 seconds, stir bar speed was $1200 \mathrm{rpm}$, and sample run time was 10 minutes after addition of $25 \mathrm{uL}$ agonist. ${ }^{13}$ Platelet aggregation (\%) was evaluated at 300s, and at maximum aggregation. Aggregation was induced using final concentrations of $1 \mu \mathrm{M}$ adenosine diphosphate (ADP), $1.5 \mathrm{mM}$ arachidonic acid (AA), $0.4 \mu \mathrm{M}$ epinephrine, and saline for spontaneous platelet aggregation. All aggregation studies were completed within 2 hours of phlebotomy, consistent with the recommendation that specimens be tested within 3-4 hours set forth by the College of American Pathologists.

Impedance Aggregometry-Multiple electrode aggregometry (Multiplate ${ }^{\mathrm{TM}}$, Dynabite, Munich, Germany) was performed in whole blood and evaluated according to manufacturer's instructions. Aggregation was induced by (final concentrations) $500 \mu \mathrm{M}$ thrombin receptor-activating peptide $32 \mu \mathrm{M}$ (TRAP) and by normal saline for spontaneous aggregation (SPA). Tests elapsed for 6 min and reported as area under the curve (AUC), maximum aggregation units (AU), and velocity.

Flow cytometry-As previously described, ${ }^{14}$ monocyte platelet aggregation (MPA) was measured via whole blood flow cytometry using the Accuri 6 system. Immediately following phlebotomy, whole blood was fixed with formaldehyde in $1.4 \times$ Hanks balanced saline solution. Blood was labeled with monoclonal antibodies CD14-PE (BD Pharmingen) and CD42a-FITC (BD Pharmingen); diluted 4.6-fold with distilled water to lyse the erythrocytes; and further diluted in an equal volume of HEPES-Tyrode's buffer, pH 7.4. Monocytes were identified by their staining with CD14-PE and by their characteristic orthogonal light scatter. The percentage of MPA was identified in single parameter histograms of CD42a-FITC fluorescence displaying events from the monocyte gate. The positive analysis region was determined using an IgG-FITC conjugated isotypic control. A minimum of 2,000 monocytes was counted per test.

Platelet activity was also assessed by activation of the platelet integrin $a \mathrm{IIb} \beta 3$ activity using a FITC-conjugated PAC-1 antibody (BD Biosciences). Platelets were labeled with anti CD42b PE antibody (BD Biosciences). The mean fluorescent intensity (MFI) was assessed within a participant gated sample of approximately 10,000 platelets. 


\section{Statistical Analysis}

Continuous variables are expressed as mean \pm SD if normally distributed and as median (25th, 75th percentile) if not. Data were compared using the Wilcoxon Signed Ranks Test for time points T1 to T4; T5 was not included in the analysis as substantially fewer samples were obtained at this time point. Specifically for MPA, missing values for T4 precluded an accurate assessment using the Wilcoxon Signed Ranks Test and as such only samples T1 to T3 were factored. Statistical significance is considered at the 5\% probability level. Calculations were performed using SPSS version 13.0 (SPSS Inc., Chicago, IL, USA).

\section{Results}

Eighteen patients undergoing open vascular surgery were included in this analysis. Mean age was $72 \pm 17$ years and $44 \%$ were female. Baseline characteristics are displayed in Table 1.

White blood cell count gradually increased over time (T1-7.9 [6.4, 9.0], T2-8.7 [6.5, 9.1], T3-9.9 [7.0, 12.1], T4-10.5 [9.4, 13.9], T5-10.3 [8.1, 13.1], $\mathrm{P}=0.001)$, whereas hematocrit decreased (T1-32.4 [29.2, 35.0], T2-31.3 [29.1, 34.5], T3-30.1 [28.6, 35.5], T4-29.4 [27.3, 35.6], T5-29.6 [25.0, 35.4], $\mathrm{P}=0.043$ ) Platelet counts were not significantly different over time (T1-198 [171-242], T2-206 [161, 237], T3-180 [142, 215], T4-168 [132, 214], T5-129 [108, 167], P=0.195) (Figure 1).

\section{Platelet Aggregation}

Spontaneous platelet aggregation increased transiently during the surgical period (T1-5.8\% [2.4, 10.8], T2-13.5\% [9.3, 26.5], T3-7.5\% [3.3, 17], T4-10.0\% [7.3, 16.3], T5-7.25\% [4.5, 29.9], $\mathrm{P}=0.002)$. Similar trends in perioperative platelet activity were noted for platelet aggregation in response to epinephrine $(\mathrm{P}=0.035)$ and $\mathrm{ADP}(\mathrm{P}=0.036$; Figure 2$)$.

\section{Platelet Activation}

Using flow cytometry, we assessed the change in MPA and PAC-1 expression - two robust markers of platelet activity. ${ }^{15,16}$ Similar to the light transmission aggregometry, there was a perioperative increase in MPA (T1-17.9 [14.7, 23.0], T2-20.1 [17.3, 27.9], T3-24.0 [19.8, 34.1], T4-19.5 [14.2, 26.1], T5-17.1 [15.1, 21.9], P=0.047), PAC-1 expression (T1-323.5 [269.8, 432], T2-416 [334.3, 607.8], T3-294 [279, 341.5], T4-347 [311, 374], T5-303 [264, 347], $\mathrm{P}=0.001$ ), and PAC-1 expression in the response to epinephrine (T1-420 [312, 687], T2-678 [444, 1361 \}, T3-415 [318, 573], T4-449 [350, 922], T5-369 [313, 491], P=0.012) (Figure 3). Both MPA and PAC-1 increased immediately during the operation, but MPA continued to rise until the third time point (1-hour post operatively). In contrast, PAC-1 had a second peak approximately 24 -hours postoperatively before returning to baseline at 48 hours.

\section{Adverse Events}

Adverse events occurring during the time of hospitalization were recorded. Among the 18 patients included, 1 patient developed a non-ST elevation myocardial infarction (NSTEMI), 1 patient who underwent lower extremity bypass developed thrombosis of his graft that 
required reoperation, and 1 patient was brought back to the operating room due to bleeding. Interestingly in the two patients with thrombotic complications, platelet activity, as assessed by MPA showed a significant increase during the perioperative period (increasing by more than $150 \%$ ) In the subject with a bleeding episode, MPA decreased by $7 \%$. A full description of all measured platelet activity markers during the perioperative period in all subjects is noted in the Supplemental table.

\section{Discussion}

This pilot study provides evidence that platelet activity increases transiently during the perioperative period in open vascular surgery. We have demonstrated that platelet activity assessed with both traditional platelet aggregation and flow cytometric techniques - is dynamic during the perioperative state. The change across different platelet assays suggests this observation reflects a real platelet phenomenon. This data may help explain the pathophysiology of increased thrombotic risk during the perioperative period of vascular surgery. ${ }^{17,18}$ This study supports the need for a better understanding of the cardiovascular (platelet medicated) risks associated with high-risk patients and an improvement in cardioprotective management, especially in the perioperative period.

Vascular surgery patients are at heightened risk for both cardiovascular and bleeding events. While antiplatelet therapy may lower cardiovascular risk preoperatively, it is frequently withheld before surgery due to the bleeding risk. A major potential benefit of understanding the platelet phenotype is that it can potentially discriminate between cardiovascular and bleeding risk along the continuum of platelet activity and hemostasis. Furthermore, platelet activity can be modified. A better understanding of platelet activity in this population may provide insight into both the mechanism of platelet mediated complications, and serve as a potential therapeutic target, thus preventing cardiovascular complications without increasing the risk of bleeding (or vice versa). Additionally, the concomitant increase in white blood cell count during the sampling period raises the question of whether the change in white blood cell count is causing the change in platelet activity, or whether both are a consequence of the same inflammatory state. Future studies by our group would be interested in further evaluating this issue.

Our study demonstrated that platelet activity increased intra-operatively and immediately following surgery with return to baseline by 48 -hours postoperatively. The use of numerous assays as well as the component of intraoperative sampling distinguishes this study from those in the past. For example, Samama et al used fewer markers and noted conflicting results; investigators found that platelet activity increased in the postoperative period at 24 and $48 \mathrm{hrs}$ by ADP-induced platelet aggregration but no change in platelet receptor expression profiling using flow cytometry. ${ }^{6}$ While Rajagopalan incorporated several markers of platelet activity (P-selectin expression, fibrinogen binding, platelet aggregation mediated by TRAP and AA), samples were only collected pre- and post-operatively; ${ }^{5}$ thus, the increase in platelet activity intra-operatively demonstrated in our data was not accessible. Another distinguishing feature of our study is the different types of vascular surgeries included. 
Several limitations need to be kept in mind when interpreting the results. As with most pilot studies, the small sample size was an important limitation in analyzing the results; and was not powered to correlate platelet activity with perioperative cardiovascular events.

Additionally, there was a significant drop in the number of samples available for testing at 48 hours post-operatively (T5) mainly due to early hospital discharge. Finally, sample collection was from the arterial line, if present, and otherwise from a venous collection. While sample processing is essential to limit sample variability, there is data suggesting good reproducibility between arterial and venous sampling. ${ }^{12,19-21}$ Recent data from our lab noted an intraclass correlation coefficient of between 0.71 to 0.76 for flow cytometric markers of platelet activity. ${ }^{12}$ Other labs noted similar levels of some, but not all, platelet activity markers in arterial and venous collections. Of note, an important correlation exists between levels of platelet activity and ex vivo processing delays in both arterial and venous collections, suggesting that sample processing can have significant influence on platelet activity markers. ${ }^{21}$ For this reason, our lab has strict methodology for platelet laboratory measurements. Despite careful attention to conditions and laboratory standardization, variability may occur. Further studies powered appropriately are needed to analyze whether perioperative platelet activity is associated with thrombotic and/or bleeding events during the perioperative period. Encouragingly, the limited number of adverse events related to thrombosis in this study did demonstrate a concomitant considerable elevation in platelet activity, though it is worth reminding that interpretation is difficult with such a small number of events. The ultimate goal is to examine perioperative platelet activity in real-time to identify patients that may be at increased risk for thrombosis and provide targeted therapeutic intervention. This will be a crucial step in the era of personalized medicine.

\section{Supplementary Material}

Refer to Web version on PubMed Central for supplementary material.

\section{Acknowledgments}

Supported in part by grant 1UL1RR029893 from the National Center for Research Resources, National Institutes of Health. Dr Berger was partially funded by an American Heart Association Fellow to Faculty Award (0775074N) and a Doris Duke Clinical Scientist Award.

\section{References}

1. Boersma E, Poldermans D, Bax JJ, Steyerberg EW, Thomson IR, Banga JD, van De Ven LL, van Urk H, Roelandt JR, Group DS. Predictors of cardiac events after major vascular surgery: Role of clinical characteristics, dobutamine echocardiography, and beta-blocker therapy. JAMA : the journal of the American Medical Association. 2001; 285:1865-1873. [PubMed: 11308400]

2. Badimon JJ, Zaman A, Helft G, Fayad Z, Fuster V. Acute coronary syndromes: pathophysiology and preventive priorities. Thrombosis and haemostasis. 1999; 82:997-1004. [PubMed: 10605815]

3. Fuster V, Badimon L, Badimon JJ, Chesebro JH. The pathogenesis of coronary artery disease and the acute coronary syndromes (1). The New England journal of medicine. 1992; 326:242-250. [PubMed: 1727977]

4. Neilipovitz DT, Bryson GL, Nichol G. The effect of perioperative aspirin therapy in peripheral vascular surgery: a decision analysis. Anesthesia and analgesia. 2001; 93:573-580. [PubMed: 11524320] 
5. Rajagopalan S, Ford I, Bachoo P, Hillis GS, Croal B, Greaves M, Brittenden J. Platelet activation, myocardial ischemic events and postoperative non-response to aspirin in patients undergoing major vascular surgery. Journal of thrombosis and haemostasis : JTH. 2007; 5:2028-2035. [PubMed: 17650080]

6. Samama CM, Thiry D, Elalamy I, Diaby M, Guillosson JJ, Kieffer E, Coriat P. Perioperative activation of hemostasis in vascular surgery patients. Anesthesiology. 2001; 94:74-78. [PubMed: 11135724]

7. Reininger CB, Reininger AJ, Steckmeier B, Greinacher A, Lasser R, Schweiberer L. Platelet response to vascular surgery--a preliminary study on the effect of aspirin and heparin. Thrombosis research. 1994; 76:79-87. [PubMed: 7817363]

8. Collins P, Ford I, Greaves M, Macaulay E, Brittenden J. Surgical revascularisation in patients with severe limb ischaemia induces a pro-thrombotic state. Platelets. 2006; 17:311-317. [PubMed: 16928603]

9. Davi G, Patrono C. Platelet activation and atherothrombosis. The New England journal of medicine. 2007; 357:2482-2494. [PubMed: 18077812]

10. Chu SG, Becker RC, Berger PB, Bhatt DL, Eikelboom JW, Konkle B, Mohler ER, Reilly MP, Berger JS. Mean platelet volume as a predictor of cardiovascular risk: a systematic review and meta-analysis. Journal of thrombosis and haemostasis : JTH. 2010; 8:148-156. [PubMed: 19691485]

11. Sharma G, Berger JS. Platelet activity and cardiovascular risk in apparently healthy individuals: a review of the data. Journal of thrombosis and thrombolysis. 2011; 32:201-208. [PubMed: 21562837]

12. Shah B, Sedlis SP, Mai X, Amoroso NS, Guo Y, Lorin JD, Berger JS. Comparison of platelet activity measurements by use of arterial and venous blood sampling. Journal of thrombosis and haemostasis : JTH. 2013; 11:1922-1924. [PubMed: 23927560]

13. Merolla M, Nardi MA, Berger JS. Centrifugation speed affects light transmission aggregometry. International journal of laboratory hematology. 2012; 34:81-85. [PubMed: 21794095]

14. Furman MI, Barnard MR, Krueger LA, Fox ML, Shilale EA, Lessard DM, Marchese P, Frelinger AL 3rd, Goldberg RJ, Michelson AD. Circulating monocyte-platelet aggregates are an early marker of acute myocardial infarction. Journal of the American College of Cardiology. 2001; 38:1002-1006. [PubMed: 11583872]

15. Freedman JE, Loscalzo J. Platelet-monocyte aggregates: bridging thrombosis and inflammation. Circulation. 2002; 105:2130-2132. [PubMed: 11994242]

16. Michelson AD, Barnard MR, Krueger LA, Valeri CR, Furman MI. Circulating monocyte-platelet aggregates are a more sensitive marker of in vivo platelet activation than platelet surface Pselectin: studies in baboons, human coronary intervention, and human acute myocardial infarction. Circulation. 2001; 104:1533-1537. [PubMed: 11571248]

17. Eagle KA, Berger PB, Calkins H, Chaitman BR, Ewy GA, Fleischmann KE, Fleisher LA, Froehlich JB, Gusberg RJ, Leppo JA, Ryan T, Schlant RC, Winters WL Jr, Gibbons RJ, Antman EM, Alpert JS, Faxon DP, Fuster V, Gregoratos G, Jacobs AK, Hiratzka LF, Russell RO, Smith SC Jr. American College of Cardiology/American Heart Association Task Force on Practice G. ACC/AHA guideline update for perioperative cardiovascular evaluation for noncardiac surgery--executive summary a report of the American College of Cardiology/American Heart Association Task Force on Practice Guidelines (Committee to Update the 1996 Guidelines on Perioperative Cardiovascular Evaluation for Noncardiac Surgery). Circulation. 2002; 105:1257-1267. [PubMed: 11889023]

18. Schneider G, Rockman C, Merolla M, Nardi M, Berger J. Platelet Activation Increases in Patients Undergoing Vascular Surgery. J Am Coll Cardiol. 2012; 59:E1699.

19. Harding SA, Din JN, Sarma J, Jessop A, Weatherall M, Fox KA, Newby DE. Flow cytometric analysis of circulating platelet-monocyte aggregates in whole blood: methodological considerations. Thrombosis and haemostasis. 2007; 98:451-456. [PubMed: 17721630]

20. Jaumdally RJ, Varma C, Blann AD, MacFadyen RJ, Lip GY. Platelet activation in coronary artery disease: intracardiac vs peripheral venous levels and the effects of angioplasty. Chest. 2007; 132:1532-1539. [PubMed: 17908707] 
21. Rondina MT, Grissom CK, Men S, Harris ES, Schwertz H, Zimmerman GA, Weyrich AS. Whole blood flow cytometry measurements of in vivo platelet activation in critically-Ill patients are influenced by variability in blood sampling techniques. Thrombosis research. 2012; 129:729-735. [PubMed: 22178064] 


\section{Highlights}

- Platelet activity increases transiently during the perioperative period in open vascular surgery

- Both platelet aggregation and platelet receptor expression profiling had similar trends

- Our data may help explain the pathophysiology of increased thrombotic risk during the perioperative period 
A. White Blood Cell Count

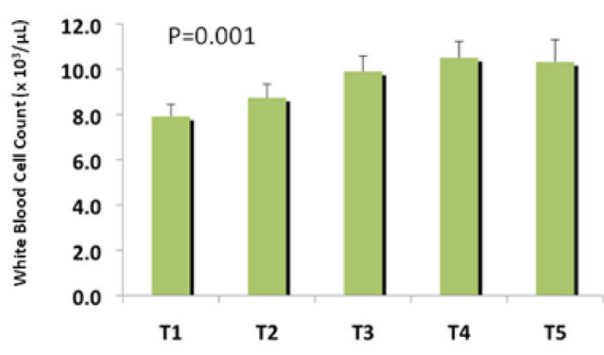

C.

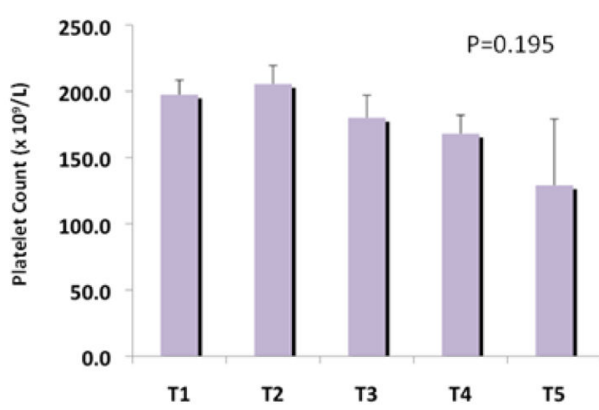

B.

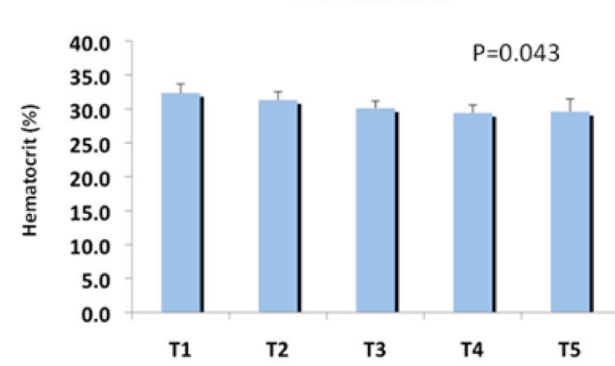

Figure 1.

Hematology analysis of the a) white blood cell count, b) hematocrit and c) platelet count during the perioperative period.

White blood cell count gradually increased with each sample, hematocrit decreased over time, and platelet count was not significantly different over time, with the only significant difference occurring between $\mathrm{T} 1$ and $\mathrm{T} 3$. 
A.

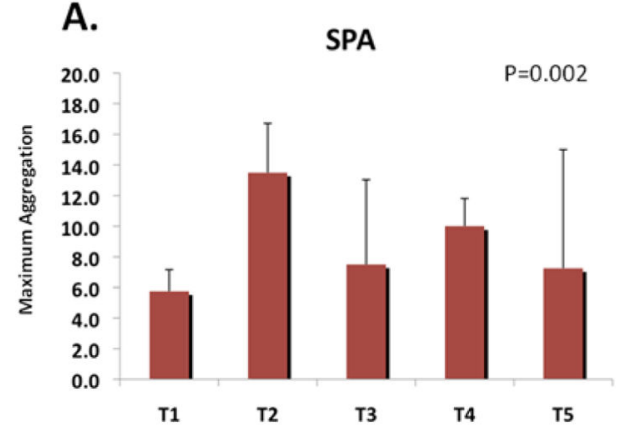

B.

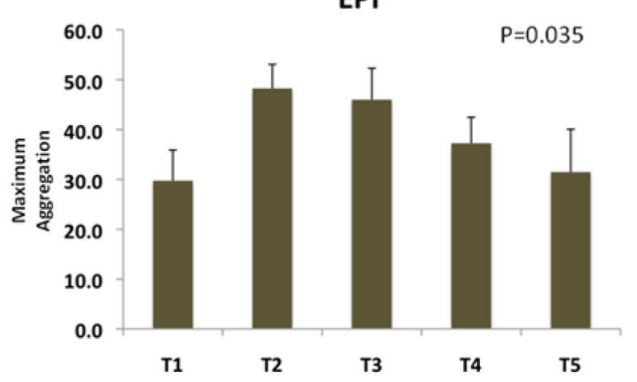

C.

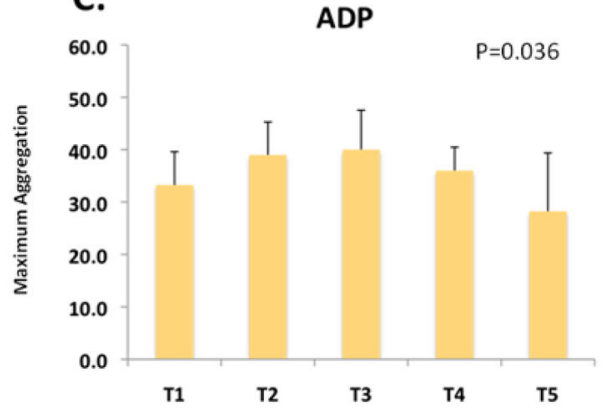

D.

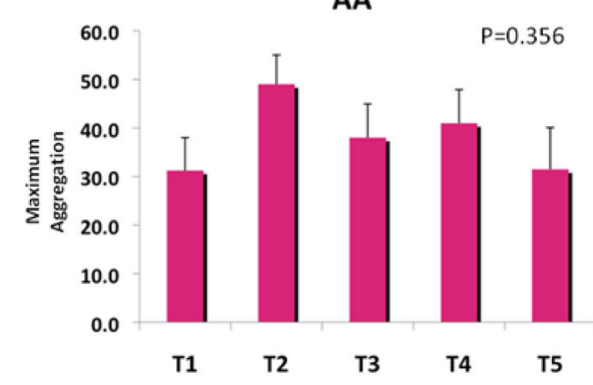

Figure 2.

Platelet aggregation in response to a) saline (spontaneous platelet aggregation), b) epinephrine, c) ADP and d) arachidonic acid during the perioperative period. Spontaneous platelet aggregation increased transiently during the surgical period. A similar transient increase was also seen in platelet aggregation in response to epinephrine, ADP and arachidonic acid. 

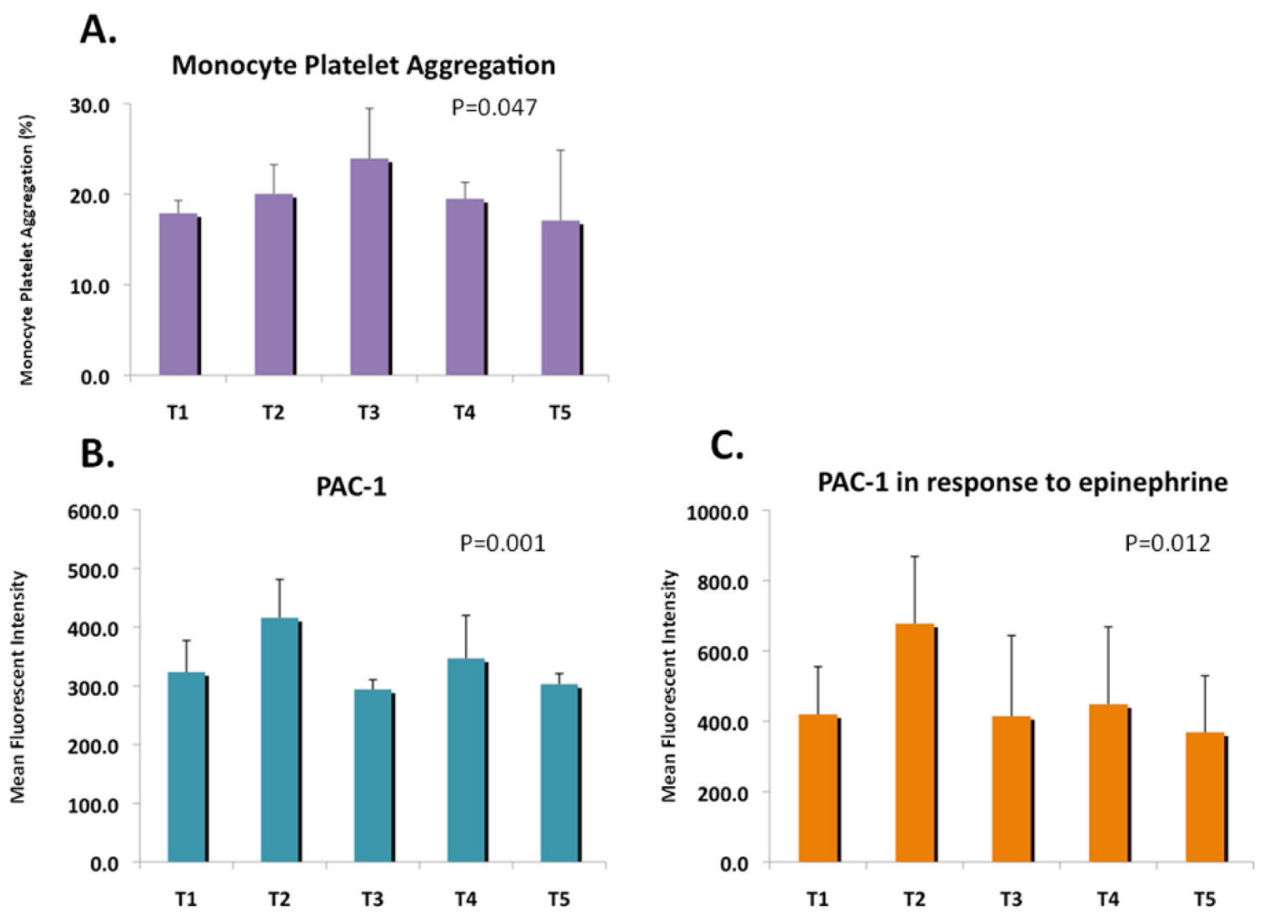

Figure 3.

Monocyte platelet aggregation, PAC-1 expression and PAC-1 expression in response to epinephrine during the perioperative period.

Monocyte platelet aggregation showed a transient increase during the perioperative period, peaking at T3. Similarly, PAC-1 expression and PAC-1 expression in response to epinephrine $0.4 \mu \mathrm{M}$ both demonstrated a significant increase perioperatively, with their peaks occurring at $\mathrm{T} 2$. 


\section{Table 1}

Preoperative Demographic and Clinical Data

\begin{tabular}{lc}
\hline & All Patients $(\mathbf{N}=\mathbf{1 8})$ No. $(\%)$ \\
\hline Age, yrs, mean \pm SD [range] & $72.0 \pm 17.0[29-94]$ \\
Gender (male/female) & $10 / 18(55.6 / 44.4)$ \\
Surgery & \\
CEA & $10(55.6)$ \\
AAA & $3(16.7)$ \\
Bypass & $5(27.8)$ \\
Smoking status & \\
Never & $7(38.9)$ \\
Current & $5(27.8)$ \\
Former & $6(33.3)$ \\
Alcohol use (drinks) & \\
None & $6(33.3)$ \\
1-6 per week & $10(55.6)$ \\
1-2 per day & $1(5.6)$ \\
3-4 per day & $1(5.6)$ \\
Use within 72 hours of surgery & $11(61.1)$ \\
Aspirin & $6(33.3)$ \\
Plavix & $14(77.8)$ \\
Statin & $1.01 \pm 0.36$ \\
Pre-op Creatinine, mean \pm SD & $109 \pm 40.5$ \\
Pre-op Glucose, mean \pm SD &
\end{tabular}

CEA, carotid endarterectomy; AAA, abdominal aortic aneurysm open repair 